\title{
External Noise Distinguishes Mechanisms of Attention
}

\author{
Zhong-Lin Lu and Barbara Anne Dosher
}

\begin{abstract}
Attention to objects or features of objects affects performance on perceptual tasks such as detection, recognition, or identification. The speed or accuracy of task performance can be significantly influenced by changes in the state of attention, especially in complex task environments. The state of attention may be manipulated by specific environmental cues, or by decisions about allocation of attention induced by task demands. The challenge is to understand and predict these attention-mediated changes in task performance, and to identify the mechanisms by which attention is operating. A theoretical and empirical framework has been developed to directly assess the mechanisms of attention by systematically manipulating the amount and/or characteristics of the external noise added to the signal stimuli and measuring modulations of perceptual discriminability (signal and noise levels) in the cognitive processes. Three classes of attention mechanisms-stimulus enhancement, external noise exclusion, and internal noise reduction, each with its signature performance pattern, can be distinguished. Empirically, the two mechanisms of stimulus enhancement and external noise exclusion are shown to occur in different circumstances. A task-taxonomy of attention mechanisms is obtained by partitioning experiments in the literature according to several factors. The framework has also been extended to the assessment of spatial and temporal characteristics of the attention window, the coordination of multimodal auditory-visual cues in the performance of visual tasks, and the action of multitask load in performance.
\end{abstract}

\section{INTRODUCTION}

Attention to objects or features of objects affects performance on perceptual tasks such as detection, recognition, or identification. The speed or accuracy of task performance can be significantly influenced by changes in the state of attention, especially in complex task environments. The question is: How does attention improve human performance? A number of functional metaphors of attention have been proposed (see LaBerge, 1995 for a review): an early sensory filter, orienting in space, a moving spotlight, a spatio-temporal gate to memory, the glue conjoining multiple features, distribution of mental resources, a control process of short-term memory. These metaphoric models of attention make strong suggestions about how attention operates, and in certain cases even admit quantitative applications. An alternative approach is to develop a formal perceptual decision structure and test models of attention effects by studying modulations of perceptual discriminability (signal and noise levels) in the cognitive processes (Dosher and Lu, 2000b; Lu and Dosher, 1998).

In the domain of signal processing, there are essentially three ways to improve the signal-to-noise ratio: amplification, improved filtering, and modified gain control. In physiology, attention has been shown to increase cellular response sensitivity (Reynolds, Pasternak, and Desimone, 2000), sharpen signal (e.g., orientation/spatial frequency) selectivity (Haenny, Maunsell, and Schiller, 1988), and to exclude unwanted information through competitive interaction, resulting in apparent shrinking of neuronal receptive fields (Desimone and Duncan, 1995). At the 
behavioral level, spatial attention has been postulated to reduce decision uncertainty (Palmer, Ames, and Lindsey, 1993), enhance the attended stimulus (Posner, Nissen, and Ogden, 1978) and/or change contrast sensitivity (Carrasco, Penpeci-Talgar, and Eckstein, 2000), exclude external noise or distractors (Shiu and Pashler, 1994), and to reduce contrast-gain control (Lee, Itti, Koch, and Braun, 1999). Motivated by neurophysiology and signal processing considerations, behavioral research based on external noise manipulations and the Perceptual Template Model (Lu and Dosher, 1998) distinguishes three mechanisms underlying performance improvements in perceptual tasks: stimulus enhancement, external noise exclusion, and multiplicative noise reduction. This review focuses on mechanisms of attention using paradigms that eliminate structural decision uncertainty; that is, observers are explicitly informed of the target location in all experimental conditions. Quantitative studies of the role of decision uncertainty, where the location of the target is not known, in paradigms involving tasks such as the classical Posner paradigm and visual search, can be found in Palmer et al. (1993).

\section{THE PERCEPTUAL TEMPLATE MODEL (PTM) APPROACH}

Observer models have been powerful tools in several areas of vision science. In the PTM approach, effects of attention are measured as a joint function of observer attention state and the amount and/or characteristics of the external noise added to the signal stimuli. Mechanisms of attention are identified as changes of observer characteristics through the perceptual template model (Dosher and Lu, 2000b; Lu and Dosher, 1998).

\section{A. Perceptual Template Model of the Observer}

Limited by various sources of noise such as intrinsic stimulus variability, receptor sampling errors, randomness of neural responses, and loss of information during neural transmission, perceptual processes exhibit various inefficiencies. Such inefficiencies at an overall system level can be modeled by characterizing perceptual processes as "perfect," noise-free computations with separate, equivalent internal noise-the amount of random internal noise necessary to produce the degree of inefficiency exhibited by the perceptual system. The PTM (Lu and Dosher, 1999) is an extension of many similar models of the human observer in the literature (Pelli, 1980).
The perceptual template model (see Fig. 74.1A) consists of five components: (1) A perceptual template with certain tuning characteristics (e.g., a spatial frequency filter $F(f)$ with a center frequency and a bandwidth such that a range of frequencies adjacent to the center frequency pass through with smaller gains). The template is normalized such that it passes the noise with gain 1.0 and the signal stimulus with gain $\beta$. (2) A nonlinear transducer function with the form output $=\|$ input $\|^{\gamma}$. (3) A multiplicative internal noise that is Gaussian distributed with mean 0 and a standard deviation that is proportional (with a coefficient of $N_{m}$ ) to the total energy in the input stimulus. Multiplicative noise is a natural way of characterizing tasks in which, for example, perceived sensory variability, or perceived differences, are proportional to signal strength (Weber-law situations). (4) An independent additive internal noise that is Gaussian distributed with mean 0 and a fixed standard deviation $N_{a}$. The existence of an absolute sensory threshold for every perceptual process suggests that the perceptual system is limited by an additive noise whose amplitude doesn't depend on the amount of input. (5) A decision process that operates on the noisy internal representation of the stimulus. Depending on the task, the decision could reflect either detection or discrimi-

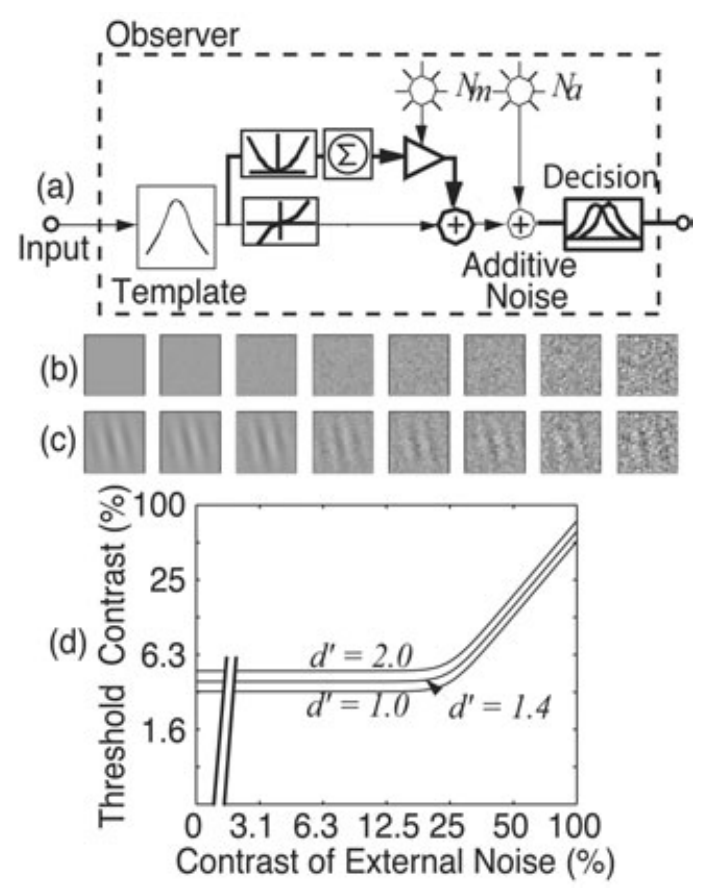

FIGURE 74.1 a. A noisy perceptual template model. b. Samples of eight levels of external noise. c. A Gabor signal embedded in the external noises shown in $\mathbf{b}$. d. Simulated threshold versus external noise contrast (TVC) functions for a perceptual template model at three criterion performance levels $\left(d^{\prime}=1.0,1.4,2.0\right)$. 
nation, and could take the form of either $\mathrm{N}$-alternative forced choice or yes/no, possibly with confidence ratings.

The observer can be characterized by systematically manipulating the amount of external noise added to the signal stimulus (see Fig. 74.1B,C) and observing how threshold-signal stimulus energy required for an observer to maintain a given performance leveldepends on the amount of external noise (the threshold versus contrast, or TVC, function; Fig. 74.1D). In a typical application, the model parameters, $N_{a}, N_{m}, \beta$, and $\gamma$, are unknown quantities that can be estimated from TVC data such as that in Fig. 74.1D by nonlinear estimation techniques, or, alternatively, simple equations can be derived that allow us to compute estimates of several of the parameters from certain relations in the data. Two or three measured threshold levels are required ( $\mathrm{Lu}$ and Dosher, 1999). Although this characterization does not distinguish between various sources for the inefficiency, it does allow us to quantify the overall efficiency of the perceptual system, and to compare the efficiency of the perceptual system in different perceptual tasks.

\section{B. The External Noise Plus Attention Paradigm and Signatures of Attention Mechanisms}

The theoretical performance signatures of attention mechanisms can be derived by studying the possible ways attention can affect various components of the PTM and generate model TVC functions for proposed attention mechanisms: stimulus enhancement, external noise exclusion, and internal multiplicative noise reduction (see Fig. 74.2).

\section{Stimulus Enhancement}

Enhanced performance due to attention in clear (noise-free) conditions corresponds to claims of perceptual enhancement (Posner et al., 1978). In the

(a) Stimulus Enhancement
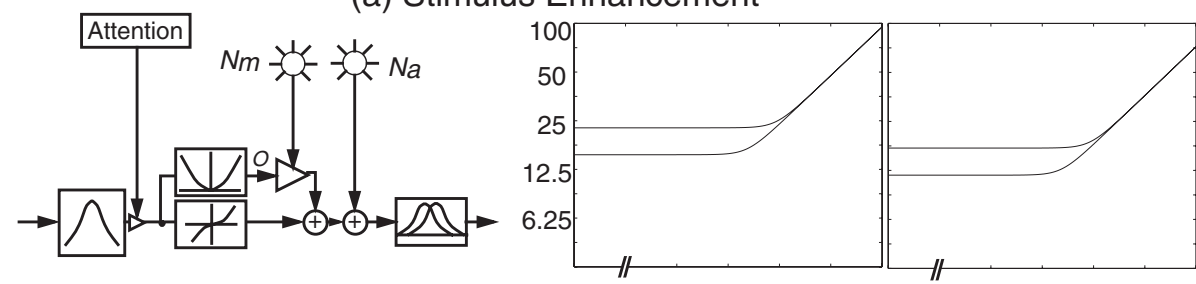

(b) External Noise Exclusion
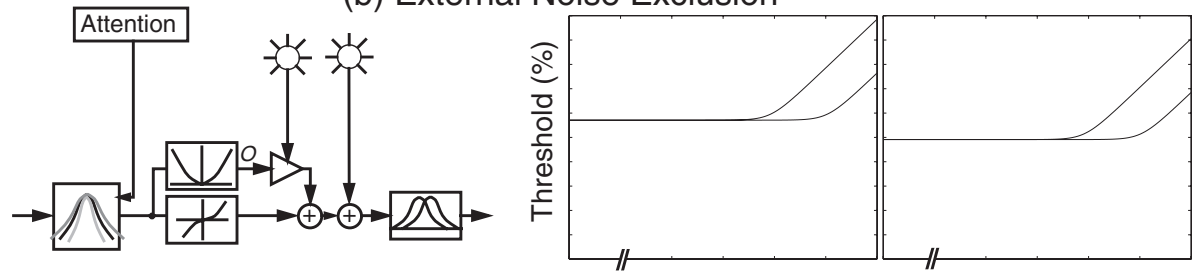

(c) Multiplicative Noise Reduction
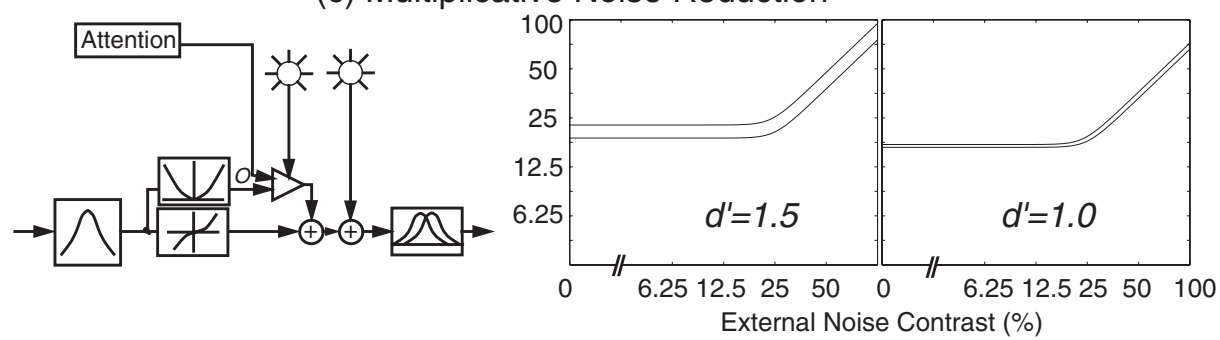

FIGURE 74.2 Signature performance patterns for three mechanisms of attention within the framework of a perceptual template model (PTM) at two performance criterion levels $\left(d^{\prime}=1.5\right.$ and 1.0). a. Stimulus enhancement. It improves performance only in zero or low external noise. b. External noise exclusion. It modulates performance only at high levels of external noise. c. Internal multiplicative noise reduction. It affects performance at all levels of external noise, but increasingly so as external noise increases. In both $\mathbf{a}$ and $\mathbf{b}$, the magnitude of attention effects does not depend on the performance criterion. In $\mathbf{c}$, however, the magnitude of the attention effects depends critically on the performance criterion. 
context of the PTM, stimulus enhancement is mathematically equivalent to internal additive noise reduction. The behavioral signature for this mechanism is performance improvement (reduced thresholds or lower curves) in the region of low or zero external noise (see Fig. 74.2A). This is because stimulus enhancement affects both the signal and the external noise in the input stimulus in the same way.

\section{External Noise Exclusion}

One key way in which attention improves performance is by focusing perceptual analysis on the appropriate time, spatial region, and/or content characteristics of the signal stimulus. This focusing serves to eliminate external noise from further processing, and is related to claims by Shiu and Pashler (1994) and others. The behavioral signature for this mechanism is performance improvements in the region of high external noise (see Fig. 74.2B), where there is external noise to exclude.

\section{Internal Noise Reduction}

Another possible mechanism of attention would involve the reduction of internal noise. The reduction of additive noise is formally equivalent to the enhancement of stimulus (see earlier). Multiplicative noise increases with increasing contrast in the stimulus display. Reduction of multiplicative internal noise produces a signature of improvements in both high and low levels of external noise, with slightly larger effects in high external noise (see Fig. 74.2C). To date, we have not empirically observed a case of multiplicative noise reduction by attention.

\section{Distinguish Mechanism Mixtures}

A direct comparison of the experimental data and the signature patterns of the PTM model may be sufficient to identify the underlying mechanism of attention in certain situations. In other situations, mixtures of more than one mechanism may underlie improvements in performance associated with attention in a task. In particular, a mixture of stimulus enhancement (low noise effects) and external noise exclusion (high noise effects) must be discriminated from multiplicative internal noise suppression (effects in low and high noise). This can be accomplished via measuring threshold versus external noise contrast at multiple criterion performance levels. A higher level of threshold performance, for example a d' of 1.5 instead of 1.0, requires higher contrast signals to achieve. For conditions differing in stimulus enhancement (see Fig. 74.2A), or in external noise exclusion (see Fig. 74.2B), the magnitude of the attention effect is the same (on the log contrast axis) at both the higher, more stringent threshold and the lower, less stringent threshold performance level. However, threshold contrast differences between two conditions at lower and higher criterion threshold values depend strongly upon criterion performance level in the conditions that differ in internal multiplicative noise reduction (see Fig. 74.2C). Thus, measuring TVC functions at two or more criterion performance levels resolves the individual contribution of each mechanism in a mixture situation (Dosher and Lu, 1999; Lu and Dosher, 1999).

\section{EMPIRICAL RESULTS AND TAXONOMY OF MECHANISMS OF SPATIAL ATTENTION}

Pure cases of stimulus enhancement ( $\mathrm{Lu}$ and Dosher, 1998; Lu, Liu, and Dosher, 2000) and external noise exclusion (Dosher and Lu, 2000a; Dosher and Lu, 2000b; Lu and Dosher, 2000; Lu, Lesmes, and Dosher, 2002) have been documented in different situations as the mechanism of location-cued visual attention. Pure external noise exclusion (see Fig. 74.2B) occurs when attention improves performance only in high noise, yet has little or no effect in conditions with low or no external noise (Dosher and Lu, 2000a; Dosher and Lu, 2000b; Lu and Dosher, 2000; Lu et al., 2002). Stimulus enhancement (see Fig. 74.2A) is demonstrated by improvements due to attention in the absence of or presence of low external noise, but cannot improve performance when it is limited by external noise ( $\mathrm{Lu}$ and Dosher, 1998; Lu et al., 2000).

Dosher and Lu (2000b) demonstrated that valid precueing one of four (or eight) widely separated spatial locations dramatically improved Gabor orientation identification relative to invalid precues in high external noise conditions. Four Gabor stimuli appeared on an annulus, one in each quadrant (see Fig. 74.3A). A $62.5 \%$ valid central arrow precue $(150 \mathrm{~ms}$ prior to the signal) indicated the likely report location. A simultaneous report cue indicated the to-be-reported target location. Full psychometric functions were measured for each external noise and attention condition. Threshold versus external noise contrast (TVC) functions at $62.5 \%$ correct is shown for the average observer in Fig. 74.3B. The primary mechanism of attention in this central cueing paradigm was external noise exclusion, implying a retuning of the perceptual template.

Two distinct attention systems have been postulated (Posner, 1980). Central cues, which point at the target location, are said to activate the endogenous attention system. Peripheral cues, which occur near the target location, are said to activate the exogenous attention 
(a)
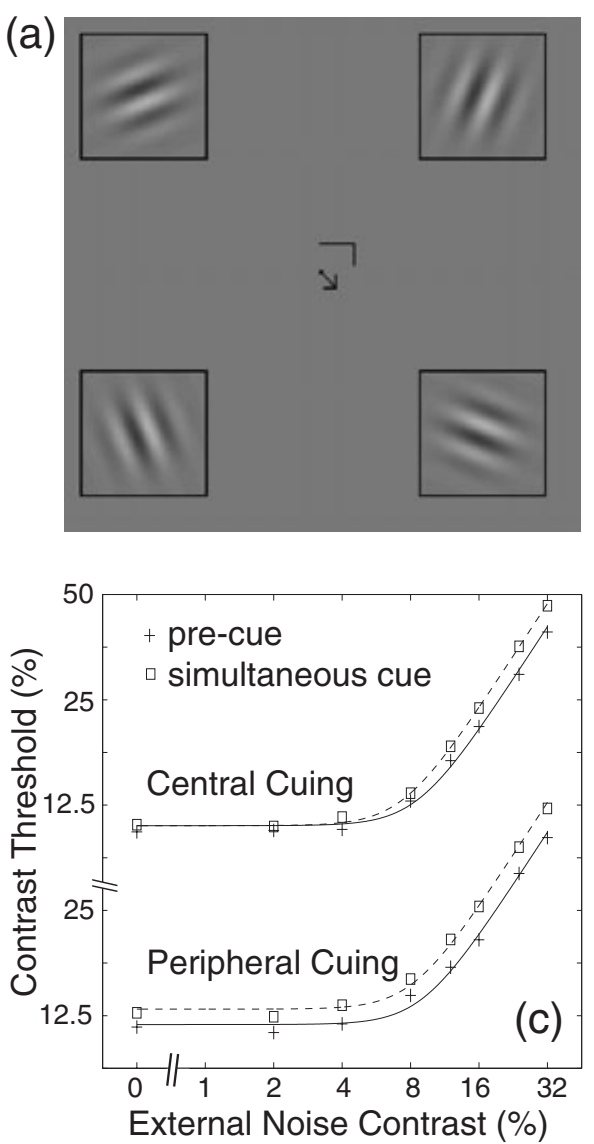
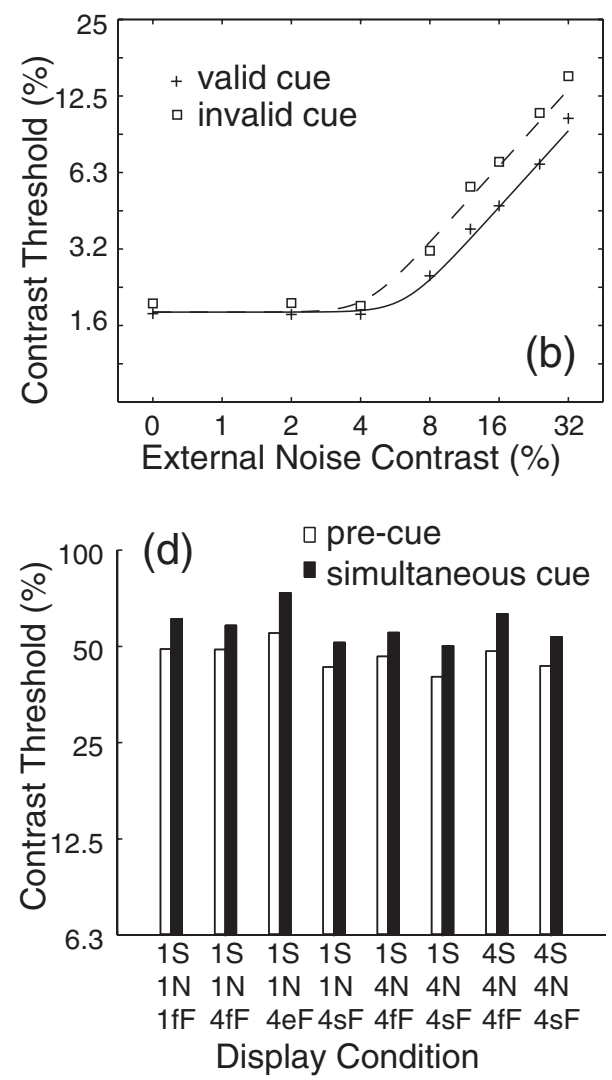

FIGURE 74.3 a. Sample layout of an invalidly cued trial. The precue (arrow) cues attention to one of four stimulus locations, and the caret cues the location to be reported (Dosher and $\mathrm{Lu}$, 2000). b. Thresholds at $62.5 \%$ correct as a function of external noise level for the average observer in the valid and invalid conditions. Cue validity has a large effect at high levels of external noise (Dosher and Lu, 2000). c. Average TVC functions at $d^{\prime}=1.24$ across all the observers in central and peripheral cuing (Lu and Dosher, 2000). In both b and c, smooth curves are best fitting PTM predictions. d. Average contrast thresholds at $62.5 \%$ correct for eight display conditions in high external noise (Lu, Lesmes, and Dosher, 2002). Denoted by jSkNltF, in each display condition, signal stimuli, external noise, and frames in $t=$ stationary, flash, or elaborated style occurred in $j=1$ or $4, k=1$ or 4 and $k \geq j$, and $l=1$ or 4 and $l \geq k$ locations.

system. The two attention systems are thought to differ in both qualitative and quantitative ways. In a new study, Lu and Dosher (2000) found that thresholds in high external noise but not zero or low external noise are improved in central precuing, whereas thresholds in both high and low external noise are improved in peripheral precuing. The results suggest that the endogenous and exogenous attention systems invoke different mechanisms of attention: external noise exclusion for the endogenous system; external noise exclusion plus stimulus enhancement for the exogenous system (see Fig. 74.3C).

Task difficulty effects associated with increasing the number of orientation templates from two to four were essentially entirely accounted for by the statistical properties of the decision task (Dosher and Lu, 2000a).
The magnitude of external noise exclusion increases with display size--external noise exclusion played little role in two-location displays; but it drastically reduced contrast thresholds in eight-location displays. Lu, Lesmes, and Dosher (2002) compared effects of precuing in a wide variety of conditions. In the absence of external noise, precuing produced only marginal performance improvements in a small random subset of display conditions; in the presence of high external noise, precuing improved task performance by the same amount in all the display conditions; and the magnitude of spatial attention effects, as gauged by contrast threshold reduction, is nearly constant across all the display conditions (see Fig. 74.3D). Furthermore, there was little or no evidence for "cross-talk" between the nontarget regions and the response in 
either the precued or the simultaneously cued conditions. The results suggest that spatial attention excludes external noise in the target region.

To summarize, external noise exclusion has been shown to be a major mechanism of spatial attention in complex multiple-location displays with either central or peripheral location cues, whereas stimulus enhancement was shown to be associated primarily with peripheral cuing of location. The magnitude of attention effects depend on the energy of external noise (masks) added to the stimuli, the number of potential target locations, and the type of (peripheral versus central) cuing. These factors, together with decision uncertainty analysis, provide an effective framework to recast and reorganize the existing literature in the area (Dosher and Lu, 2000a).

\section{OTHER APPLICATIONS AND EXTENSIONS}

The PTM approach has recently been applied to identify the mechanisms of performance improvements in perceptual learning, object-based attention, and cross-modal cuing of spatial attention. The PTM model and the external noise paradigm have also been further extended to characterize the tuning characteristics of the perceptual template in spatial frequency, space, and time under attended and unattended states. These and other studies will further clarify the functional nature of external noise exclusion, and provide important links to results from neurophysiology.

\section{Acknowledgment}

This research was supported by US Air Force Office of Scientific Research, Visual Information Processing Program.

\section{References}

Carrasco, M., Penpeci-Talgar, C., and Eckstein, M. (2000). Spatial covert attention increases contrast sensitivity across the CSF: Support for signal enhancement. Vision Research 40, 1203-1215.
Desimone, R., and Duncan, J. (1995). Neural mechanisms of selective visual attention. Annual Review of Neuroscience 18, 193-222.

Dosher, B. A., and Lu, Z.-L. (1999). Mechanisms of perceptual learning. Vision Research 39, 3197-3221.

Dosher, B. A., and Lu, Z.-L. (2000a). Mechanisms of perceptual attention in precuing of location. Vision Research 40, 1269-1292.

Dosher, B. A., and Lu, Z.-L. (2000b). Noise exclusion in spatial attention. Psychological Science 11, 139-146.

Haenny, P. E., Maunsell, J. H. R., and Schiller, P. H. (1988). State dependent activity in monkey visual cortex. Experimental Brain Research 69, 245-259.

LaBerge, D. (1995). Attentional processing: The brain's art of mindfulness. Perspectives in cognitive neuroscience (pp. x, 262). Harvard University Press, Cambridge, MA, US.

Lee, D. K., Itti, L., Koch, C., and Braun, J. (1999). Attention activates winner-take-all competition among visual filters. Nature Neuroscience 2, 375-381.

Lu, Z.-L., and Dosher, B. A. (1998). External noise distinguishes attention mechanisms. Vision Research 38, 1183-1198.

Lu, Z.-L., and Dosher, B. A. (1999). Characterizing human perceptual inefficiencies with equivalent internal noise. Journal of the Optical Society of America A Special Issue: Noise in imaging systems and human vision 16, 764-778.

Lu, Z.-L., and Dosher, B. A. (2000). Spatial attention: Different mechanisms for central and peripheral temporal precues? Journal of Experimental Psychology: Human Perception and Performance 26, 1534-1548.

Lu, Z.-L., Lesmes, L. A., and Dosher, B. A. (2002). Spatial attention excludes external noise at the target location. Journal of Vision 2, 312-323.

Lu, Z.-L., Liu, C. Q., and Dosher, B. A. (2000). Attention mechanisms for multi-location first- and second-order motion perception. Vision Research 40, 173-186.

Palmer, J., Ames, C. T., and Lindsey, D. T. (1993). Measuring the effect of attention on simple visual search. Journal of Experimental Psychology: Human Perception \& Performance 19, 108-130.

Pelli, D. G. (1980). Effects of visual noise. (Ph. D. dissertation) University of Cambridge, Cambridge, England.

Posner, M. I., Nissen, M. J., and Ogden, W. C. (1978). Attended and unattended processing modes: The role for spatial location. In "Modes of perceiving and processing information" (N. H. L. Pick and I. J. Saltzman Eds.), (pp. 137-157). Erlbaum, Hillsdale, NJ.

Reynolds, J. H., Pasternak, T., and Desimone, R. (2000). Attention increases sensivity of V4 neurons. Neuron 26, 703-714.

Shiu, L., and Pashler, H. (1994). Negligible effect of spatial precuing on identification of single digits. Journal of Experimental Psychology: Human Perception and Performance 20, 1037-1054. 